\title{
A structure hierarchy for chain-, ribbon- and tube-silicate minerals: a bond topological approach
}

Maxwell C. Day and Frank C. Hawthorne

University of Manitoba, C.H.R. Faculty of Environment, Earth and Resources

A structure hierarchy is a classification of atomic arrangements ranked according to their principal constituent-cation polyhedra and the connectivity of those polyhedra. Such hierarchies (1) provide a framework to aid in the understanding of factors controlling composition and structural variability of minerals, and (2) help link different crystallization mechanisms with particular chemical compositions and structural arrangements. To date, structure hierarchies have been developed for mineral classes such as phosphates, vanadates, borates and arsenates. However, a coherent structure hierarchy has yet to be developed for silicate minerals, despite their abundance in the Earth's crust. The proposed structure hierarchy organizes chain-, ribbon- and tube-silicates based on (1) the type and degree of $\left[\mathrm{SiO}_{4}\right]^{4-}$ linkages, and (2) the connectivity and geometry of tetrahedra within the irreducible length (repeat-unit) of each chain. Each structure is grouped into first- and second-order divisions based on the O:Si ratio and the connectedness of tetrahedra, respectively. In an effort to more easily determine and graphically illustrate variables (1) and (2), each chain of tetrahedra is represented as a graph, where tetrahedra are reduced to vertices (points) and linkage between tetrahedra to edges (lines).

By sequentially inserting 1-, 2-, 3- and 4-connected vertices in a graph, all algebraically possible graph (chain) arrangements can be generated, which in turn allows for the comparison of chains that occur with those that do not occur in minerals. Of the $\sim 440$ chain-silicate minerals, 375 correspond to only four topologically unique graphs. To understand these apparent controls on chain-silicate stability, additional properties must be examined. One such property is complexity or information entropy, which refers to the amount of information (in bits) contained within the repeat-unit of the chain of interest. Calculating the complexity of every graph shows that, in general, chain-silicate minerals with higher abundance correspond to graphs of lower complexity, suggesting that complexity may influence chain-silicate stability. 